\title{
MELHORIA NO RENDIMENTO DE FERRO-LIGAS E DOS PROCESSOS DE DESOXIDAÇÃO E DESSULFURAÇÃO COM A UTILIZAÇÃO DE CARBURETO DE CÁLCIO*
}

\author{
Lívia Lopes de Oliveira Goulart ${ }^{1}$ \\ André Luiz Vasconcellos da Costa e Silva ${ }^{2}$ \\ Ely da Silva Araujo Junior ${ }^{3}$
}

\begin{abstract}
Resumo
Visando garantir o nível de qualidade dos produtos e o controle do processamento do aço para atender aos requisitos dos clientes e das normas técnicas aplicáveis e manter-se competitivo no mercado de aços longos com redução contínua de custos, um dos grandes desafios das aciarias elétricas é promover o aumento do rendimento de suas ferro-ligas melhorando ao mesmo tempo os processos de desoxidação e dessulfuração, além da limpeza interna do banho. Este trabalho teve como objetivo avaliar o efeito da adição de carbureto de cálcio durante o vazamento das corridas do aço 1026-D nestas variáveis, através da análise estatística e termodinâmica de dados de corridas experimentais, com e sem adição de carbureto de cálcio. Foi demonstrado que a adição desse insumo melhora de forma significativa os parâmetros metalúrgicos de qualidade do material e reduz o custo de transformação para a fabricação desse produto através da redução do consumo de ferro-ligas.
\end{abstract}

Palavras-chave: Desoxidação; Dessulfuração; Carbureto de cálcio; Ferro-ligas.

\section{IMPROVEMENT OF ALLOYS-IRON'S YIELD AND THE DEOXIDATION AND DESULPHURIZATION PROCESSES WITH THE CALCIUM CARBIDE USE}

\section{Abstract}

Aiming to guarantee the quality level of products and steel processing control to meet customers' requirements and standards further applicable rules, remain competitive in the long steel market aiming ongoing cost reduction, one of the higher challenge on electric steelworks is promoting alloy iron yield improve, improving the same time the deoxidation and desulphurization processes thus the metallic internal cleansing. This work aimed measure the gains talked regarding the addition of calcium carbide during the tapping of 1026-D steel, through experimental data reviews in statistic and through a mass balance evaluation did for analyze the alloys iron yields. Was demonstrated that the calcium carbide addition improve, significantly, the material quality metallurgical parameters and decrease the transformation cost for product fabrication through alloy iron consume decrease.

Keywords: Deoxidation; Desulphurization; Calcium carbide; Alloys-iron.

1 Engenheira Metalúrgica, Eng. de Processos da Aciaria Elétrica, VSBM, Barra Mansa, RJ, Brasil; livia.goulart.lg1@vsiderurgia.com.br.

2 Engenheiro Metalúrgico, PhD, Professor, EEIMVR, UFF, Membro da ABM, Volta Redonda, RJ, Brasil; andre@metal.eeimvr.uff.br.

3 Engenheiro Metalurgista; M.Eng., Gerente da Aciaria Elétrica, VSBM, Resende, RJ, Brasil; ely.araujo@vsiderurgia.com.br. 


\section{INTRODUÇÃO}

Desoxidação e dessulfuração são duas etapas importantes para a qualidade dos aços longos, com grande influência no custo do processo.

A oxidação excessiva do aço reduz o rendimento da maior parte das ligas adicionadas no momento do vazamento. Além disto, quanto mais oxigênio estiver dissolvido no aço e tiver de ser removido através destas reações, tanto maior será a formação de inclusões não-metálicas, influenciado a limpeza interna do aço produzido. As perdas de ferro, de elementos de liga adicionados e o consumo dos desoxidantes impactam no custo de fabricação do produto final.

Quanto mais bem desoxidado estiver o banho, tanto melhor será a dessulfuração, para uma mesma escória. A composição química do aço deve estar bem ajustada para que, após a dessulfuração, exista Mn suficiente para combinar com o $\mathrm{S}$ que resta no metal, através da formação do MnS, evitando a formação do FeS, de baixo ponto de fusão, fonte potencial de fragilidade a quente. A relação $\mathrm{Mn} / \mathrm{S}$ também influencia a ocorrência da romboidade, (defeito físico em que o tarugo tem diagonais de dimensões diferentes) no lingotamento contínuo. Assim, uma melhor dessulfuração, além de contribuir para diminuir a formação de sulfetos de baixo ponto de fusão, evita o gasto excessivo de Mn para atingir um mesmo valor de $\mathrm{Mn} / \mathrm{S}$, já que o valor final de $\mathrm{S}$ é reduzido.

A influência direta na qualidade (através do teor de enxofre, da limpeza interna, por exemplo) e nos custos (através do rendimento de ferro-ligas e desoxidantes) indica que as etapas citadas merecem cuidados especiais. Uma adição que pode contribuir tanto para a desoxidação como para a dessulfuração do aço com custo competitivo é o $\mathrm{CaC}_{2}$, especialmente quando aços de ultra-baixo e baixo carbono não são objetivados.

O presente trabalho propõe avaliar o efeito da adição de $\mathrm{CaC}_{2}$ no vazamento das corridas no FEA sobre a desoxidação do banho, rendimento das ferro-ligas e eficiência da dessulfuração. Além disso, o projeto tem como objetivo avaliar o efeito destas adições, combinadas com as práticas de desoxidação presentemente utilizadas na Siderúrgica Barra Mansa, sobre a partição de S entre metal e escória e formação de sulfetos de baixo ponto de fusão, pela análise da relação $\mathrm{Mn} / \mathrm{S}$ e limpeza interna através de análise de inclusões.

\subsection{Desoxidação do Banho Metálico}

A desoxidação é uma etapa importante na fabricação do aço. Essa etapa visa principalmente:

- Diminuição do teor de O dissolvido no aço, evitando a formação de FeO, de baixo ponto de fusão, ou a formação de poros de gás, nucleados pela formação de CO;

- Redução dos óxidos FeO e MnO da escória, visando melhor rendimento de ligas oxidáveis a serem adicionadas;

- Garantir o rendimento, com reprodutibilidade, das ligas adicionadas ao banho;

- Tornar o ambiente da panela redutor após o vazamento do FEA, para favorecer o processo de dessulfuração no Forno Panela (FP).

A escolha dos desoxidantes recai sobre os elementos formadores de óxidos mais estáveis e compatíveis com o aço. 
De acordo com Turkdogan [1], três elementos satisfazem as condições acima de forma mais eficaz na siderurgia. São eles:

- Manganês utilizado na forma de ferro-ligas (alto ou baixo carbono);

- Silício como composto ou ferro-liga ou adicionado juntamente ao Mn na forma de FeSiMn;

- Alumínio.

Além dos desoxidantes citados acima, $\circ \mathrm{CaC}_{2}$ também tem sido avaliado pelo seu alto potencial de dessulfuração e desoxidação. Há diferentes práticas de desoxidação adotadas em diferentes usinas. A sequência de adição dos desoxidantes, por exemplo, ainda é assunto de discussão. Segundo Ribeiro [2], a desoxidação começa com a injeção do desoxidante de menor poder seguida pela adição de um desoxidante mais forte, agitando sempre o banho para que ocorra a flotação dos óxidos formados para a escória. Assim, os dados termodinâmicos recomendariam a adição do $\mathrm{Mn}$ seguida pelo Si e pelo $\mathrm{CaC}_{2}$. Porém, não há consenso com relação a melhor sequência. Em primeiro lugar, a desoxidação simultânea com Mn e Si pode aproveitar melhor a formação dos óxidos compostos destes elementos, que reduzem ainda mais o oxigênio dissolvido. Assim, é até bastante comum observar nas siderúrgicas que o desoxidante que forma o óxido de maior estabilidade é adicionado primeiramente no banho metálico, assim como proposto por Bruch [3]. Essa é a prática que será utilizada nesse trabalho.

\subsection{Dessulfuração do Banho Metálico}

Um dos procedimentos mais importantes que segue a desoxidação do aço é sua dessulfuração. Na maior parte dos aços fabricados (excluindo aqueles destinados à usinagem), o S é um elemento residual indesejado seja pela formação de sulfetos que podem causar falha a quente no tratamento termomecânico dos aços. $O$ controle deste fenômeno, através da adição de Mn pode aumentar a quantidade de inclusões no produto final, e por esses motivos, o $S$ normalmente é mantido em níveis baixos no aço. A desufuração consiste na passagem do enxofre do banho metálico para a escória e ocorre sob condições desoxidantes, ou seja, com mínimo de $O$ dissolvido no aço, possível, como mostra a reação 1.

A reação de dessulfuração em ambientes redutores é dada por:

$$
[\mathrm{S}]+\mathrm{O}^{2-}=[\mathrm{O}]+\mathrm{S}^{2-}
$$

Para manter condições desoxidantes, as escórias devem, também, ser bem desoxidadas, ou seja, seus teores de $\mathrm{FeO}$ e $\mathrm{MnO}$ devem ser bastante baixos para que o oxigênio que pode ser dissolvido no banho por meio do equilíbrio com estes óxidos (reoxidação) não prejudique a dessulfuração. Por outro lado, a adição de $\mathrm{CaC}_{2}$ pode promover escória favorável para ocorrência da dessulfuração, já que torna os teores de FeO e MnO menores e aumenta a basicidade da mesma.

Uma forma simplificada de avaliar a dessulfuração, é através da razão de partição do enxofre. Esta é a relação entre a concentração de enxofre na escória e a do metal, no equilíbrio, dada pela equação 2 .

$$
\mathrm{L}_{\mathrm{S}}=\frac{(\% S)}{[\% S]}
$$

A partição $\left(L_{S}\right)$, no entanto somente poderá ser comparada entre escórias e entre usinas, se o valor de [O] for mantido constante, uma vez que a retirada de $S$ do banho depende do potencial do oxigênio, como visto acima. 


\subsection{Desoxidação e Dessulfuração com Carbureto de Cálcio}

$\mathrm{O} \mathrm{CaC}_{2}$ promove a desoxidação do banho a partir da formação de produtos de desoxidação sólidos e gasosos. O produto gasoso se dá com a desoxidação através do $\mathrm{C}$, dando origem ao gás CO. De acordo com Maia e colaboradores [4], essa desoxidação pode ser profundamente alterada por variações na pressão desse gás. A reação de desoxidação é dada abaixo:

$$
\mathrm{C}+\mathrm{O}=\mathrm{CO}
$$

Além disso, $\mathrm{o} \mathrm{CaC}_{2}$ promove a desoxidação com produto sólido dando origem ao $\mathrm{CaO}$. Nesse caso, a desoxidação ocorre através da interação do $\mathrm{O}$ dissolvido no banho e também através dos óxidos mais redutíveis presentes como inclusões no banho ou na escória. Esses óxidos são $\mathrm{FeO}$ e $\mathrm{MnO}$. Escórias com alto teor de $\mathrm{FeO}$ têm alto potencial de oxigênio e, consequentemente, elevado teor valor de $\mathrm{MnO}$, o que causa baixa recuperação de ferro-ligas, desgaste excessivo dos refratários na linha de escória, além de dessulfuração deficiente. A desoxidação com $\mathrm{CaC}_{2}$ pode reverter essa situação porque, como atua como desoxidante, diminui as atividades dos óxidos formados, melhorando a recuperação das ferro-ligas como pode ser visto nas reações de desoxidação com produto sólido abaixo:

$$
\begin{aligned}
\mathrm{CaC} 2+3(\mathrm{FeO}) & \rightarrow(\mathrm{CaO})+2 \mathrm{CO}+3[\mathrm{Fe}] \\
\mathrm{CaC} 2+3(\mathrm{MnO}) & \rightarrow(\mathrm{CaO})+2 \mathrm{CO}+3[\mathrm{Mn}]
\end{aligned}
$$

Campos e colaboradores [5] confirmam que a dessulfuração na panela está diretamente ligada à boa desoxidação do aço. Além disso, a absorção de $\mathrm{S}$ é influenciada pela viscosidade da escória, 0 que influencia a cinética de dessulfuração. A adição de $\mathrm{CaC}_{2}$ favorece a desoxidação e aumenta $\mathrm{B}_{2}$ pela formação de $\mathrm{CaO}$. A presença de $\mathrm{Ca}$ dissolvido no aço pode, também afetar a morfologia das inclusões. Como a cinética é muito importante, a eficiência do tratamento em FP na desulfuração e promoção da limpeza interna etapa dependerá também do tempo de permanência da corrida no FP, determinante para a flotação de inclusões e sua captação pela escória.

\section{MATERIAL E MÉTODOS}

\subsection{Material}

O material estudado foi o aço 1026-D, produzido pela usina Votorantim Siderurgia unidade Barra Mansa. Esse material é destinado à laminação de vergalhões de diferentes bitolas. Esse grau de aço foi escolhido por conta de sua elevada escala de produção, aproximadamente $300 \mathrm{kt}$ anuais, sendo seu processo bastante representativo no que diz respeito às variáveis metalúrgicas e custo da aciaria em geral.

\subsection{Métodos}

Foram acompanhadas corridas deste aço durante 3 meses. Avaliou-se o consumo de ferro-ligas, a partição do enxofre entre metal e escória e o efeito da adição de diferentes quantidades de $\mathrm{CaC}_{2}$ durante o vazamento do FEA para a panela.

Este estudo foi dividido em duas etapas: Primeiro foram realizadas medidas de composição química de escória e aço na entrada e saída no FP e composição 
química de aço no lingotamento contínuo, além de valores de $\mathrm{B}_{2}, \% \mathrm{FeO}+\mathrm{MnO}$ e do consumo de ferro-ligas. Para caracterizar o processo de dessulfuração, as variáveis observadas foram partição de $S$ na saída do FP e relação $M n / S$ das amostras da MLC. A segunda etapa envolveu um balanço de massa para avaliar o rendimento das ferro-ligas levando em conta a adição ou não do carbureto de cálcio durante o vazamento das corridas no FEA. Por fim, buscou-se correlações estatísticas entre as variáveis medidas e os resultados dos balanços de massa dos elementos de liga.

As corridas foram comparadas segundo a quantidade de $\mathrm{CaC}_{2}{ }^{1}$ adicionadas no momento do vazamento do FEA para a panela. As quantidades foram 0, 45 e $60 \mathrm{~kg}$.

\subsubsection{Balanço de massa para estimativa do rendimento das ferro-ligas}

Como parte do trabalho, visou-se construir um modelo que fosse capaz de prever, com base em um balanço de massa, o rendimento de Si e Mn a partir da adição ou não de $\mathrm{CaC}_{2}$ no aço.

A base para a construção do modelo foram cálculos estequiométricos, levando em conta a formação dos óxidos de Si, Mn, e Ca durante a desoxidação do aço e da escória.

O balanço de massa feito estava diretamente relacionado com a oxidação do banho, que foi calculada em cada caso pela quantidade de $O$ dissolvida. Uma relação empírica exponencial entre PPM $\mathrm{O}$ dissolvido no aço medido por célula eletroquímica e o teor de $\mathrm{FeO}$ na escória obtido por análise química foi obtida através de análises de escória do FEA e foi realizada uma comparação do \%MnO para cada \%FeO amostrado, dando origem à tabela abaixo (os dados foram extrapolados até $\quad 1200$ PPM O).

Tabela 1: Valores de \%FeO e \%MnO obtidos através de regressão com o valor de PPM O aço.

\begin{tabular}{c|c|c|c}
\hline PPM O & $\%$ FeO & \% MnO & $\begin{array}{c}\% \text { FeO + \% } \\
\text { MnO }\end{array}$ \\
\hline 200 & 19,17 & 5,92 & 25,09 \\
\hline 250 & 20,06 & 4,61 & 24,67 \\
\hline 300 & 20,98 & 5,86 & 26,84 \\
\hline 350 & 21,94 & 6,08 & 28,02 \\
\hline 400 & 22,95 & 5,74 & 28,69 \\
\hline 450 & 24,01 & 6,18 & 30,19 \\
\hline
\end{tabular}

Foi levada em conta a quantidade de Ferro-ligas utilizada no vazamento das corridas, calculando a contribuição da desoxidação feita através da adição de Si e Mn. Para cálculo da quantidade final de latas de carbureto de cálcio a serem adicionadas, foi estabelecido que, de acordo com Ribeiro [2], um nível de oxidação aceitável para aços "não especiais" é de 30 PPM de O no aço, e um percentual de 1,5 de FeO+MnO para a escória de saída do FP.

\footnotetext{
${ }^{1}$ Produto fornecido pela White Martins, denominado Carbureto de Cálcio com $75 \%$ de $\mathrm{CaC}_{2}$.
} 


\section{RESULTADOS E DISCUSSÃO}

\subsection{Resultados}

\subsubsection{Rendimento de ligas}

Os rendimentos de $\mathrm{Si}$ e $\mathrm{Mn}$ foram avaliados através de análise dos dados experimentais coletados e do balanço de massa citado na seção 2.2.1. Três situações foram tratadas separadamente:

- Situação 1: Cálculo da quantidade de $\mathrm{CaC}_{2}$ negativa (indica que foi utilizado desoxidante em excesso, não sendo necessária a adição de $\mathrm{CaC}_{2}$ );

- Situação 2: Quantidade de $\mathrm{CaC}_{2}$ positiva, porém sem adição do insumo;

- Situação 3: Quantidade de $\mathrm{CaC}_{2}$ positiva, com adição do insumo.

Os resultados de rendimento das ligas foram avaliados quanto às diferenças entre métodos em teste de hipóteses com 95\% de confiança. Os resultados obtidos são apresentados na tabela 2 .

Tabela 2: Resultados de rendimento de Si e Mn para os três métodos utilizados no balanço de massa.

\begin{tabular}{c|c|c|c}
\hline \multicolumn{4}{|c}{ Rendimento de Ligas (\%) } \\
\hline \multirow{2}{*}{ Liga } & \multicolumn{3}{|c}{ Métodos } \\
\cline { 2 - 4 } & 1 & 2 & 3 \\
\hline $\mathrm{Si}$ & 44 & 48,11 & 53,25 \\
\hline $\mathrm{Mn}$ & 105,93 & 109,64 & 109,64 \\
\hline
\end{tabular}

Da tabela 2, percebe-se que os ganhos em rendimento de Si e Mn foram maiores na situação 3. Os demais parâmetros de oxidação, dessulfuração e limpeza interna do banho foram comparados de acordo com a quantidade de $\mathrm{CaC}_{2}$ adicionada, sendo elas, 0, 45 e $60 \mathrm{~kg}$.

\subsubsection{Desoxidação do sistema aço/escória}

Assumindo uma boa agitação e contato metal-escória-adicões durante o vazamento, pode-se supor que, no momento de chegada ao Forno Panela (FP), aço e escória estejam bem próximos do equilíbrio: desta forma, menores teores de \%FeO+\%MnO indicam aço mais bem desoxidado. A tabela 3 apresenta os valores medidos da soma \%FeO+\%MnO na chegada ao FP, em função da quantidade de carbureto de cálcio adicionado no vazamento do FEA.

Tabela 3: \%FeO+\%MnO de chegada no FP em função da quantidade de carbureto de cálcio adicionado.

\begin{tabular}{cc}
\hline $\begin{array}{c}\text { Carbureto de } \\
\text { Cálcio }(\mathrm{kg})\end{array}$ & $\% \mathrm{FeO+ \% MnO}$ \\
\hline 0 & 6,69 \\
\hline 45 & 2,49 \\
\hline 60 & 1,7 \\
\hline
\end{tabular}




\subsubsection{Dessulfuração do banho metálico}

Assumindo que para ocorrer uma dessulfuração eficaz no FP necessita-se de, além de outros fatores, ambiente redutor (redução do nível de oxidação aço/escória) e basicidade binária $\left(B_{2}\right)$ elevada, na tabela 4 , percebe-se outra contribuição da adição de $\mathrm{CaC}_{2}$ no banho metálico.

Tabela 4: $\mathrm{B}_{2}$ de chegada no FP em função da quantidade de adição de $\mathrm{CaC}_{2}$.

\begin{tabular}{cc}
\hline $\begin{array}{c}\text { Carbureto de } \\
\text { Cálcio }(\mathrm{kg})\end{array}$ & $\begin{array}{c}\text { B2 de } \\
\text { chegada no } \\
\text { FP }\end{array}$ \\
\hline 0 & 1,44 \\
\hline 45 & 1,72 \\
\hline 60 & 1,67 \\
\hline
\end{tabular}

A partir de melhor desoxidação e $\mathrm{B} 2 \mathrm{com}$ adição de $\mathrm{CaC}_{2}$, observa-se, na Tabela 5 um aumento na partição de enxofre (Ls), constatando uma melhor dessulfuração.

Tabela 5: Partição de $S$ entre escória e aço no $\mathrm{FP}$, em função da quantidade de adição de $\mathrm{CaC}_{2}$.

\begin{tabular}{cc}
\hline $\begin{array}{c}\text { Carbureto de } \\
\text { Cálcio }(\mathrm{kg})\end{array}$ & $\begin{array}{c}\text { Ls de saída } \\
\text { do FP }\end{array}$ \\
\hline 0 & 4,42 \\
\hline 45 & 7,62 \\
\hline 60 & 14,35 \\
\hline
\end{tabular}

\subsection{Discussão}

\subsubsection{Rendimento de ligas}

Como observado na tabela 2, o rendimento das ligas (elementos Si e Mn) aumentou quando foi utilizado $\mathrm{CaC}_{2}$ no momento do vazamento da corrida do FEA para a panela. Esse aumento no rendimento das ligas é devido a contribuição do $\mathrm{CaC}_{2}$ para a desoxidação do aço e da escória. Esta contribuição ocorre diretamente (via reação do $\mathrm{CaC}_{2}$ com o oxigênio dissolvido e com o $\mathrm{FeO}$ e $\mathrm{MnO}$ da escória) como através do efeito favorável da $\mathrm{CaO}$ formada, que reduz a atividade da $\mathrm{SiO}_{2}$ na escória, principal produto de desoxidação na prática adotada na VSBM.

Os ganhos obtidos em adição de ferro-ligas na situação 3 foram comparados com as outras situações, sendo $11,65 \%$ menor que o utilizado na situação 1 e $5 \%$ menor quando comparado a situação 2.

\subsubsection{Desoxidação do sistema aço/escória}

Em relação ao \%FeO+MnO, notou-se na tabela 3 que quando $60 \mathrm{~kg}$ de $\mathrm{CaC}_{2}$ foi adicionado, as corridas chegavam mais desoxidadas no FP que quando $45 \mathrm{~kg}$ foram utilizados. Esse resultado já era esperado uma vez que quanto mais desoxidante é usado, menor deve ser a quantidade de óxidos redutíveis presentes na escória. Essa afirmativa pode ser reforçada a partir da análise do gráfico da figura 1. 


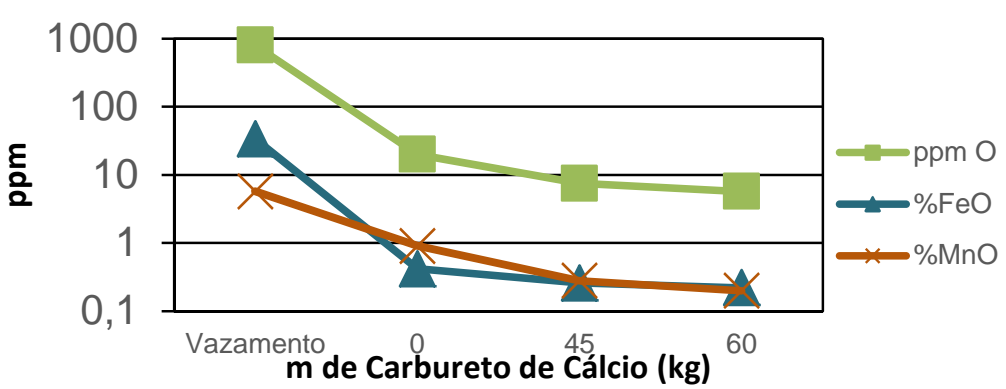

Figura 1: Relação entre ppm O no aço, \% FeO e \%MnO na escória calculado pelo programa Thermocalc.

Esse gráfico foi calculado pelo programa Thermo-calc, considerando $\mathrm{T}=1600^{\circ} \mathrm{C} \mathrm{e}$ que o equilíbrio foi atingido. O valor calculado difere um pouco dos dados experimentais, uma vez que foram consideradas situações onde o equilíbrio termodinâmico foi atingido. Os resultados, são, porém, consistentes com o resultado experimentais encontrados.

\subsubsection{Dessulfuração do banho metálico}

A Tabela 4 indica que a basicidade binária aumenta com a adição de $\mathrm{CaC}_{2}$, favorecendo a dessulfuração. Porém, percebeu-se que com $45 \mathrm{~kg}$ B2 foi maior que com $60 \mathrm{~kg}$ de adição. Essa variação pode ter ocorrido devido a agitação do banho no momento de retirada da escória, não permitindo que toda a $\% \mathrm{SiO}_{2}$ tivesse tempo de flotar para a escória com $45 \mathrm{~kg}$ de $\mathrm{CaC}_{2}$, evidenciando uma maior $\mathrm{B}_{2}$.

Foi mostrado o aumento na partição de $S$, o que sugere que uma dessulfuração mais eficaz aconteceu com a adição de $\mathrm{CaC}_{2}$. Os resultados experimentais estão de acordo com o calculado pelo Thermo-calc, mostrando que adicionando $60 \mathrm{~kg}$ de CaC2, Ls praticamente dobra de valor (Figura 2).

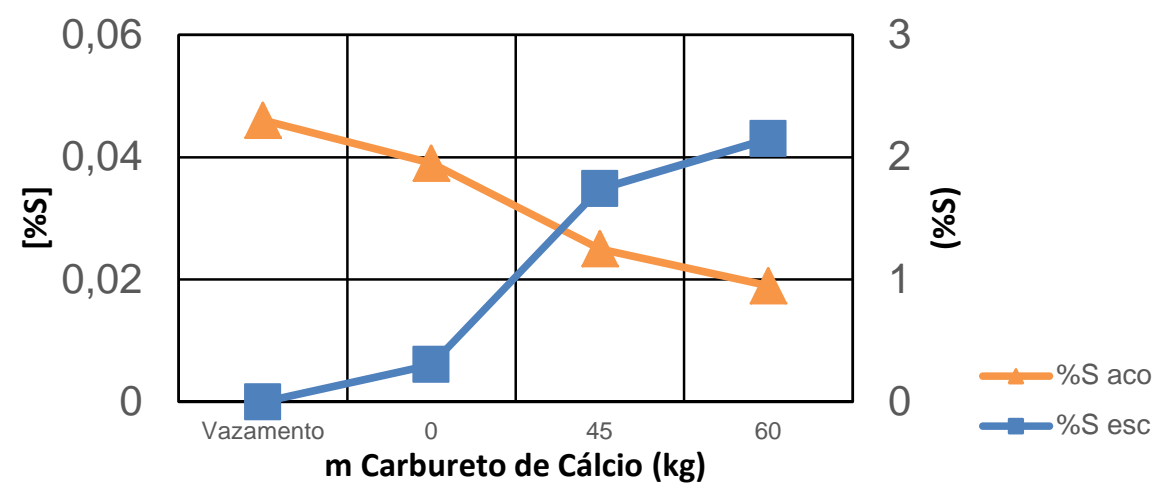

Figura 2: Relação entre as quantidades de $\mathrm{S}$ na escória e no aço com a adição de $\mathrm{CaC}_{2}$.

Com uma melhor dessulfuração, é possível garantir uma maior relação $\mathrm{Mn} / \mathrm{S}$ com uma menor quantidade de Mn adicionado, evitando a formação de FeS e fragilidade a quente e reduzindo o potencial para formação de romboidade durante 0 lingotamento. Para o aço 1026-D, o mínimo requerido para $\mathrm{Mn} / \mathrm{S}$ é 20. Essa disponibilidade de economia de ferro-ligas a base de Mn pode ser evidenciada na tabela 6. 
Tabela 6: Comparação entre rel. $\mathrm{Mn} / \mathrm{S}$ e \%Mn adicionado em função da adição de $\mathrm{CaC}_{2}$.

\begin{tabular}{ccc}
\hline $\begin{array}{c}\text { Carbureto de } \\
\text { Cálcio }(\mathrm{kg})\end{array}$ & Rel. Mn/S & $\% \mathrm{Mn}$ \\
\hline 0 & 26 & 0,67 \\
\hline 45 & 27 & 0,66 \\
\hline 60 & 28 & 0,64 \\
\hline
\end{tabular}

A limpeza interna do banho evidenciada pela menor formação de inclusões pode ser observada na tabela 7 que mostra a quantidade de inclusões ASTM A analisadas em todos os campos dos tipos A, B, C e D.

Tabela 7: Inclusões ASTM A Thin e Heavy de 0 a 2. Tipos (a) A; (b) B; (c) C; (d) D.

\begin{tabular}{c|cccc}
\hline \multicolumn{1}{c}{ Type A } & Okg & $45 \mathrm{~kg}$ & $60 \mathrm{~kg}$ \\
\hline \multirow{2}{*}{0} & Thin & 63,5 & 52,15 & 53,5 \\
\cline { 2 - 5 } & Heavy & 73 & 52,25 & 54,25 \\
\hline \multirow{2}{*}{0,5} & Thin & 7,5 & 0,25 & 0,75 \\
\cline { 2 - 5 } & Heavy & 1 & 0,25 & 0,25 \\
\hline \multirow{2}{*}{1} & Thin & 3 & 0 & 0,5 \\
\cline { 2 - 5 } & Heavy & 0 & 0 & 0,5 \\
\hline \multirow{2}{*}{1,5} & Thin & 0,5 & 0,15 & 0,25 \\
\hline & Heavy & 0,5 & 0 & 0 \\
\hline \multirow{2}{*}{2} & Thin & 0 & 0 & 0 \\
\cline { 2 - 5 } & Heavy & 0 & 0 & 0 \\
\hline
\end{tabular}

(a)

\begin{tabular}{c|cccc}
\hline \multicolumn{2}{c}{ Type C } & $0 \mathrm{~kg}$ & $45 \mathrm{~kg}$ & $60 \mathrm{~kg}$ \\
\hline \multirow{2}{*}{0} & Thin & 66,5 & 52,5 & 53,25 \\
\cline { 2 - 5 } & Heavy & 72 & 52,25 & 54,6 \\
\hline \multirow{2}{*}{0,5} & Thin & 5,5 & 0 & 1,75 \\
\cline { 2 - 5 } & Heavy & 1,5 & 0,25 & 0 \\
\hline \multirow{2}{*}{1} & Thin & 2 & 0 & 0 \\
\cline { 2 - 5 } & Heavy & 1 & 0 & 0,4 \\
\hline \multirow{2}{*}{1,5} & Thin & 1 & 0 & 0 \\
\cline { 2 - 5 } & Heavy & 0 & 0 & 0 \\
\hline \multirow{2}{*}{2} & Thin & 0 & 0 & 0 \\
\cline { 2 - 5 } & Heavy & 0,5 & 0 & 0 \\
\hline
\end{tabular}

(c)

\begin{tabular}{c|cccc}
\hline & Type B & $0 \mathrm{~kg}$ & $45 \mathrm{~kg}$ & $60 \mathrm{~kg}$ \\
\hline \multirow{2}{*}{0} & Thin & 72,5 & 52,5 & 54,6 \\
\cline { 2 - 5 } & Heavy & 75 & 52,5 & 55 \\
\hline \multirow{2}{*}{0,5} & Thin & 2,5 & 0 & 0,15 \\
\cline { 2 - 5 } & Heavy & 0 & 0 & 0 \\
\hline \multirow{2}{*}{1} & Thin & 0 & 0 & 0,25 \\
\cline { 2 - 5 } & Heavy & 0 & 0 & 0 \\
\hline \multirow{2}{*}{1,5} & Thin & 0 & 0 & 0 \\
\cline { 2 - 5 } & Heavy & 0 & 0 & 0 \\
\hline \multirow{2}{*}{2} & Thin & 0 & 0 & 0 \\
\cline { 2 - 5 } & Heavy & 0 & 0 & 0 \\
\hline
\end{tabular}

(b)

\begin{tabular}{c|cccc}
\hline \multicolumn{2}{c}{ Type D } & $0 \mathrm{~kg}$ & $45 \mathrm{~kg}$ & $60 \mathrm{~kg}$ \\
\hline \multirow{2}{*}{0} & Thin & 58,5 & 52,5 & 45,25 \\
\cline { 2 - 5 } & Heavy & 72,5 & 52,4 & 52,65 \\
\hline \multirow{2}{*}{0,5} & Thin & 10 & 0 & 7,85 \\
\cline { 2 - 5 } & Heavy & 2,5 & 0,15 & 2,35 \\
\hline \multirow{2}{*}{1} & Thin & 2,5 & 0 & 1,9 \\
\cline { 2 - 5 } & Heavy & 0 & 0 & 0 \\
\hline \multirow{2}{*}{1,5} & Thin & 3,5 & 0 & 0 \\
\cline { 2 - 5 } & Heavy & 0 & 0 & 0 \\
\hline \multirow{2}{*}{2} & Thin & 0,5 & 0 & 0 \\
\cline { 2 - 5 } & Heavy & 0 & 0 & 0 \\
\hline
\end{tabular}

(d)

A menor quantidade de inclusões se deu quando foi adicionado $\mathrm{CaC}_{2}$. O resultado é esperado pela melhor desoxidação, desulfuração e eventual efeito globulizador de inclusões no FP. 


\section{CONCLUSÕES}

Comprovou-se que a adição de $\mathrm{CaC}_{2}$ melhora a desoxidação e dessulfuração, além do rendimento das ligas e limpeza interna do aço 1026-D fabricado pela aciaria elétrica da VSBM. Em detalhe, as observações foram:

- O melhor resultado de rendimento de Si se deu na situação 3 (adição de CaC2) enquanto o pior resultado ocorreu na situação 1 (desoxidação não requer adição de $\mathrm{CaC}_{2}$.)

- Os ganhos representaram redução de $5 \%$ de consumo de ferro-ligas no método 3 em relação ao 2 e 11,95\% no método 3 em relação ao 1;

- A desoxidação das corridas durante o vazamento do FEA até sua chegada no FP foi expressivamente melhor quando se utilizou $\mathrm{CaC}_{2}$ como desoxidante, sendo \%FeO+MnO na escória mais baixo quando a adição ocorreu;

- A dessulfuração também foi efetiva com a utilização de $\mathrm{CaC}_{2}$, o que permitiu garantir Mn/S satisfatório de forma a diminuir a formação de sulfetos de baixo ponto de fusão com uma adição menor de $\mathrm{Mn}$ com $60 \mathrm{~kg}$ de $\mathrm{CaC}_{2}$, reduzindo os gastos com as ferro-ligas;

A quantidade de $\mathrm{CaC}_{2}$ a ser adicionada no vazamento pode ser de $45 \mathrm{~kg}$ ou $60 \mathrm{~kg}$, uma vez que ambas promoveram melhor rendimento e menor consumo de ferroligas, atendendo simultaneamente aos parâmetros de desoxidação e dessulfuração do banho, garantindo a limpeza interna de sulfetos de baixo ponto de fusão. A adição de $60 \mathrm{~kg}$, entretendo, garantiu resultados melhores que a de $45 \mathrm{~kg}$.

\section{Agradecimentos}

À Escola de EEIMVR e à Votorantim Siderurgia Barra Mansa pelo apoio e suporte técnico. Ao INT, Rio de Janeiro, RJ pelo apoio e análises realizadas.

\section{REFERÊNCIAS}

1 E. T. Turkdogan and G. J. W. Kor - Sulfides and Oxides in Fe-Mn alloys: part I. Phase relations in Fe-Mn-S-O system, Metallurgical Transactions, 2(1971) 1561-1570.

2 D. B. Ribeiro - Curso de Refino Secundário dos Aços, ABM, 2010.

3 R. Bruch. Estudo termodinâmico da desoxidação do aço e escória com a adição de carbureto de cálcio, UFRS, Porto Alegre, 2006.

4 B. T. Maia, F. L. Monteiro, G. Menezes, H. G. de Souza e N. J. de Carvalho. Implantação da desoxidação do aço com uso de CaC2. XXXVII Seminário de Aciaria ABM, Porto Alegre, 2006.

5 A. A. Campos, A. B. Lana, E. S. Cavalieri, J. D. G. A. Júnior, J. E. Pinto, J. E. Ank, N. S. Ferreira, O. A. C. Teixeira e R. Martins. Desoxidação e dessulfuração dos aços carbono e ligados com a utilização de CaC2. XXXIX Seminário de Aciaria ABM, Curitiba, 2008. 